\title{
Long noncoding RNA Braveheart promotes cardiogenic differentiation of mesenchymal stem cells in vitro
}

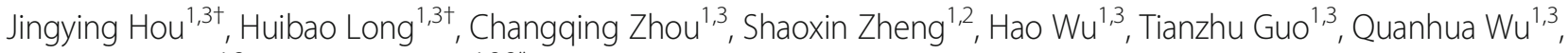
Tingting Zhong ${ }^{1,3}$ and Tong Wang ${ }^{1,2,3^{*}}$

\begin{abstract}
Background: Mesenchymal stem cells (MSCs) have limited potential of cardiogenic differentiation. In this study, we investigated the influence of long noncoding RNA Braveheart (IncRNA-Bvht) on cardiogenic differentiation of MSCs in vitro.

Methods: MSCs were obtained from C57BL/6 mice and cultured in vitro. Cells were divided into three groups: blank control, null vector control, and IncRNA-Bvht. All three groups experienced exposure to hypoxia $\left(1 \% \mathrm{O}_{2}\right)$ and serum deprivation for $24 \mathrm{~h}$, and $24 \mathrm{~h}$ of reoxygenation $\left(20 \% \mathrm{O}_{2}\right)$. Cardiogenic differentiation was induced using 5-AZA for another $24 \mathrm{~h}$. Normoxia $\left(20 \% \mathrm{O}_{2}\right)$ was applied as a negative control during the whole process.

Cardiogenic differentiation was assessed, and expressions of cardiac-specific transcription factors and epithelialmesenchymal transition (EMT)-associated biomarkers were detected. Anti-mesoderm posterior1 (Mesp1) siRNA was transfected in order to block its expression, and relevant downstream molecules were examined.

Results: Compared with the blank control and null vector control groups, the IncRNA-Bvht group presented a higher percentage of differentiated cells of the cardiogenic phenotype in vitro both under the normal condition and after hypoxia/re-oxygenation. There was an increased level of cTnT and a-SA, and cardiac-specific transcription factors including Nkx2.5, Gata4, Gata6, and Isl-1 were significantly upregulated $(P<0.01)$. Expressions of EMT-associated genes including Snail, Twist and N-cadherin were much higher $(P<0.01)$. Mesp1 exhibited a distinct augmentation following IncRNA-Bvht transfection. Expressions of relevant cardiac-specific transcription factors and EMT-associated genes all presented a converse alteration in the condition of Mesp1 inhibition prior to IncRNA-Bvht transfection.

Conclusion: IncRNA-Bvht could efficiently promote MSCs transdifferentation into cells with the cardiogenic phenotype in vitro. It might function via enhancing the expressions of cardiac-specific transcription factors and EMT-associated genes. Mesp1 could be a pivotal intermediary in the procedure.
\end{abstract}

Keywords: Long noncoding RNA Braveheart, Mesenchymal stem cells, Cardiogenic differentiation, Cardiac specific transcription factors, Epithelial-mesenchymal transition, Mesoderm posterior1

\footnotetext{
*Correspondence: tongwang316@163.com

${ }^{\dagger}$ Equal contributors

'Guangdong Provincial Key Laboratory of Malignant Tumor Epigenetics and

Gene Regulation, the Sun Yat-sen Memorial Hospital of Sun Yat-sen

University, 107 Yanjiang Xi Road, Guangzhou, Guangdong 510120, China

${ }^{2}$ Guangdong Province Key Laboratory of Arrhythmia and Electrophysiology,

107 Yanjiang Xi Road, Guangzhou, Guangdong 510120, China

Full list of author information is available at the end of the article
} 


\section{Background}

Cardiovascular disease remains a major cause of morbidity and mortality worldwide [1]. The current treatment options for end-stage heart failure fail to regenerate myocardium that has gone through necrosis or apoptosis. Induction of cardiac regeneration to replace the lost cardiomyocytes in the injured heart represents a promising therapeutic approach in this context [2]. Stem cell therapy has emerged as a novel strategy for the treatment of ischemic heart disease during the past decade. Various stem cell types have been used for the repair of the damaged heart [2-4]. Noteworthy benefits are revealed in the regeneration of cardiomocytes following the transplantation of the precursor cells [2-4]. However, the underlying molecular mechanisms that lead to cardiomyocyte regeneration after cell therapy have not been fully elucidated.

Bone marrow-derived mesenchymal stem cells (BMMSCs) have a great potential of proliferation and differentiation, and they have been considered as a suitable source for cell therapy $[5,6]$. Mesenchymal stem cells (MSCs) are capable of differentiating into cardiomyocytes under appropriate conditions both in vitro and in vivo [6]. In spite of this, the transdifferentiation efficiency of these cells is extremely low. Currently, several measures have been developed to promote the differentiation of MSCs into cardiomyocytes [7, 8]. However, most of these methods are inefficient and only a small percentage of differentiated cells can be produced. How to gain a high rate of cardiogenic differentiation from MSCs has become an issue that needs to be addressed.

Stem cell transdifferentiation into cardiomyocytes fundamentally relies on elaborate cellular and molecular mechanisms [9]. Recent discoveries demonstrate that the non-coding portion of the genome plays a crucial role in controlling cellular fate, phenotype and behavior [10]. A large number of noncoding RNAs (ncRNAs) that function as central orchestrators of cell-specific gene networks have been identified [10,11]. An important subclass of these ncRNAs is the long noncoding RNAs (lncRNAs) that are broadly defined as regulatory noncoding transcripts more than 200 nucleotides in length. Although their biological roles and mechanisms of function remain largely elusive, accumulating evidence shows that lncRNAs participate in a wide spectrum of biological processes including cellular development, disease etiology, stem cell pluripotency and lineage specification [12]. There are already a handful reports indicating that lncRNAs can modulate cardiac differentiation during heart development [13, 14]. The long noncoding RNA Braveheart (lncRNA-Bvht) is a heartassociated lncRNA that has been identified as a pivotal regulator of cardiac lineage specification and differentiation [14]. It mediates cardiac commitment epigenetically and performs critical roles during cardiac differentiation in mouse embryonic stem cells (ESCs).
Epithelial-mesenchymal transition (EMT) is a biological process that is implicated in the developmental stage, organogenesis, tissue repair and pathological conditions [15]. Emerging evidence indicates that EMT might result in transformation of stem cell phenotypes. EMT accompanies transitions between stem-like cells and their more differentiated progeny, which perform critical functions in tissue repair and regeneration [16]. It has been revealed that EMT is involved in cardiac differentiation of ESCs and pluripotent stem cells (PSCs) [17, 18].

Mesoderm posterior 1 (Mesp1) is an essential transcription factor that marks a common multipotent cardiovascular progenitor [14]. Its expression can induce cardiovascular progenitor cells [19]. lncRNA-Bvht functions via Mesp1 to modulate the expression of cardiac transcription factors and further promote cardiogenic differentiation of ESCs [14]. Previous data show that Mesp1 is capable of initiating the EMT process by regulating EMT-associated genes [20].

In this study, lncRNA-Bvht was transfected into MSCs of $\mathrm{C} 57 \mathrm{BL} / 6$ mice in order to investigate its implication on cardiogenic differentiation of these cells, and the underlying mechanism involved were explored in the procedure.

\section{Methods}

\section{Ethics statement}

Three-week-old C57BL/6 mice were obtained from the Animal Experimental Center of the Sun Yat-sen University. All animal handling and procedures were performed in accordance with protocols approved by the Animal Ethics Committee of Sun Yat-sen University (201210016).

\section{Isolation and culture of bone marrow-derived mesenchymal} stem cells

All experiment protocols described were approved by the Institutional Animal Care \& Use Committee (IACUC) at Sun Yat-sen University. Bone marrow cells were collected from 3 to 4 weeks old C57BL/6 mice by flushing femurs and tibias under aseptic conditions. Cells were cultured $\left(37^{\circ} \mathrm{C}, 5 \% \mathrm{CO}_{2}\right)$ in $25 \mathrm{~cm}^{2}$ culture flasks with complete culture medium supplemented with $10 \%$ fetal bovine serum, L-glutamine $(4.0 \mathrm{mM})$, penicillin $(100 \mathrm{IU} / \mathrm{mL})$ and streptomycin $(100 \mu \mathrm{g} / \mathrm{mL})$. On the third day of culture, the medium was replaced and non-adherent cells were removed. The adherent cells were washed two times gently with phosphate-buffered saline (PBS) to reduce the degree of hematopoietic lineage cell contamination. The cells were cultured in complete culture medium and the medium was changed every 3 to 4 days for 3-4 weeks. Adherent cells gaining 90\% confluence were trypsinized with $0.25 \%$ trypsin-ethylenediamine tetraacetic acid (Invitrogen) and passaged into new flasks for 
further expansion. Characteristics of MSCs were identified by fluorescence-activated cell sorting as previously reported [21].

\section{IncRNA-Bvht vector construction}

The pre-lncRNA-Bvht oligonucleotides were chemically synthesized by Jinweizhi Co. Ltd. (Jiangsu, China). The primers were as follows: Xhol forward: $5^{\prime}$ ccgCTCGAG GATCTCTGCCCCTCAGAGTCC3', BamHI reverse: 5' cgcGGATCCAACATTTATTTTTAAAGTTTA 3'. The recovered polymerase chain reaction (PCR) products with the precursor sequence for lncRNA-Bvht were inserted into pLVX-IRES-ZsGreen1 vector. After the pre-lncRNA-Bvht viral-based vector was transformed to DH5 $\alpha$ cells, antibiotic-resistant colonies were selected on LB-ampicillin $(100 \mu \mathrm{g} / \mathrm{mL})$ agar plates. The plasmid containing the target gene was verified by PCR, double digestion and DNA sequencing.

\section{IncRNA-Bvht transfection}

The monolayer of MSCs of uniform growth attaining $90 \%$ confluence were passaged. Culture medium was removed and cells were trypsinized with $0.25 \%$ trypsinethylenediamine tetraacetic acid (Invitrogen). The cells were re-seeded at a density of $1 \times 10^{6}$ cells per cell culture flask with complete medium for $24 \mathrm{~h}$. Cells gaining 70-80\% confluence were applied for transfection. The pLVX-IRES-ZsGreen1 vector encoding IncRNA-Bvht was transfected into MSCs with lipofectamine 2000 (Invitrogen) according to the manufacturer's instructions. The medium was changed with fresh complete DMEM $8 \mathrm{~h}$ after transfection. The expression of ZsGreen was checked after $48 \mathrm{~h}$ of transfection.

\section{siRNAs experiments}

MSCs were incubated at $1 \times 10^{6}$ cells per well in six-well plates at day 0 with siRNAs against Mesp1 (Sigma) or control siRNAs (negative control, NC; Sigma). Transfection of siRNAs was performed using lipofectamine 2000 (Invitrogen) according to the manufacturer's instructions. Mesp1 knockdown was determined by quantitative real-time PCR.

\section{Hypoxia/reoxygenation treatment of MSCs}

MSCs in the blank control, null vector control and lncRNA-Bvht groups all experienced hypoxia/reoxygenation treatment. Cells in the different groups were incubated in serum-free media with $1 \% \mathrm{O}_{2}$ in a Galaxy ${ }^{\circ}$ $48 \mathrm{R}$ incubator (Eppendorf/Galaxy Corporation, USA) at $37{ }^{\circ} \mathrm{C}$ for $24 \mathrm{~h}$ and exposed to normoxic condition $\left(20 \% \mathrm{O}_{2}\right)$ for another $24 \mathrm{~h}$. Normoxia was used as a negative control during the experiments for the three groups.

\section{Cardiogenic differentiation of MSCs}

Differentiation of MSCs to cardiogenic cells was accomplished afterwards. MSCs of the three groups were seeded into six-well plates at a concentration of $1 \times 10^{6}$ cells per well. To induce cell differentiation, the cells were incubated in a medium containing 5-AZA (10uM; Sigma-Aldrich) for $24 \mathrm{~h}$ at $37{ }^{\circ} \mathrm{C}$ in a humidified atmosphere with $5 \% \mathrm{CO}_{2}$. The cells were then washed twice and the medium was replaced with normal DMEM. The medium was changed every 3 days and this procedure was terminated at 2 weeks. The morphological changes in MSCs were observed under a microscope (Olympus, CX41).

\section{Immunofluorescence staining}

Slides with the treated cell samples taken from dishes were used directly. After drying at room temperature for a few minutes, they were permeabilized in $2 \%$ formaldehyde/PBS for $10 \mathrm{~min}$. Antigen retrieval was followed by microwaving sections in sodium citrate buffer $(1 \mathrm{M}$, $\mathrm{pH}$ 6.1). Sections were blocked with $5 \%$ bovine serum albumin (BSA) at room temperature before incubating with primary antibodies at $4{ }^{\circ} \mathrm{C}$ overnight (dilution cTnT, 1:100; $\alpha$-SA, 1:100). After washing, sections were incubated with appropriate secondary antibodies and slides were counterstained with 4-6-diamidino-2-phenylindole (DAPI). Images were taken by fluorescent microscopy (Leica, Germany) with a CCD camera (Tokyo, Japan). The percentage of cTnT-positive cells was used to evaluate the efficiency of MSCs transdifferentiated into cells with the cardiogenic phenotype.

\section{Western blot analysis}

Protein levels were measured by western blot. Cells were washed several times with PBS before collection and lysed with modified RIPA buffer. Cells were completely lysed after repeated vortexing, and supernatants were acquired though centrifugation at $14,000 \times g$ for $20 \mathrm{~min}$. Proteins were resolved by sodium dodecyl sulfatepolyacrylamide gel (SDS-PAGE) and transferred to a polyvinylidenedifluoride (PVDF) membrane (IPVH00010, Millpore, Boston, USA) before incubation with the primary antibodies overnight at $4{ }^{\circ} \mathrm{C}$. The membranes were subjected to three 5-min washes with TBST and incubated with anti-IgG horseradish peroxidase-conjugated secondary antibody (Southern biotech, Birmingham, USA) for $60 \mathrm{~min}$ at room temperature. After extensive washing, bands were detected by enhanced chemiluminescence. The band intensities were quantified by using image software (image J $2 \times$, version 2.1.4.7).

\section{Quantitative real-time PCR}

Total RNA was isolated from cells using a Trizol reagent (Invitrogen) followed by digestion with RNase-free DNase 
(Promega). Concentration and integrity of total RNA were estimated and the real-time PCR was conducted on an ABI PRISM 7500 Sequence Detection System using SYBR Green qPCR SuperMix (Invitrogen). The primers are described in Table 1. Specific products were amplified and detected at $95{ }^{\circ} \mathrm{C}$ for $10 \mathrm{~min}$, followed by 40 cycles at $95{ }^{\circ} \mathrm{C}$ for $15 \mathrm{~s}$ and at $60{ }^{\circ} \mathrm{C}$ for $30 \mathrm{~s}$, at which point data were acquired. The relative level of mRNA was calculated using the $2^{-\Delta \Delta \mathrm{Ct}}$ method. For the assays of the molecules examined, the results were quantified as the threshold cycle of each target gene and normalized into $\Delta \mathrm{Ct}$ value. Quantifications of fold-change in gene expressions were also performed using the $2^{-\Delta \Delta \mathrm{Ct}}$ method.

\section{Statistical analysis}

All quantitative data are described as mean $\pm \mathrm{SD}$. The significance of differences among groups was determined by the analysis of variance and Scheffe's multiplecomparison techniques. Comparisons between time-based measurements within each group were performed with analysis of variance for repeated measurements. A $P$ value $<0.05$ was considered to be statistically significant.

\section{Results}

PCR amplification and sequencing of IncRNA-Bvht

DNA fragments of lncRNA-Bvht were successfully amplified by PCR. Electrophoresis revealed the specific band of lncRNA-Bvht at $500 \mathrm{bp}$ (Fig. 1A). The sequence of lncRNA-Bvht was analyzed (Fig. 1B).

\section{IncRNA-Bvht transfection efficiency}

ZsGreen was expressed after MSCs were transduced with the pLVX-IRES-ZsGreen1 vector. All the MSCs with ZsGreen expression were observed under the microscope (Fig. 2A). After IncRNA-Bvht tranfection, its expression in different cell groups was detected by quantitative real-time PCR. The mRNA level was significantly higher in the lncRNA-Bvht group compared with the blank control and null vector control groups (Fig. 2B; $P<0.01$ ).

\section{Cardiogenic differentiation in different cell groups}

Cardiogenic differentiation in different cell groups was examined by immunofluorescence staining (Fig. 3). Morphology changes could be observed in different cells groups after 14 days of induction. The differentiated MSCs expressed cardiomyocyte-specific cell markers including cTnT (green colour; Fig. 3A1-A6, images b) and $\alpha$-SA (red colour; Fig. 3A1-A6, images c). The lncRNABvht group (Fig. 3A3 and A6) showed an obviously higher percentage of cTnT-positive cells than the blank control (Fig. 3A1 and A4) and null vector control groups (Fig. 3A2 and A5) both under the normal condition and after the hypoxia/reoxygenation treatment $(P<0.01$; Fig. 3A1-A6 and B); mRNA and protein levels of cTnT and $\alpha$-SA were also elevated after lncRNA-Bvht transfection (Fig. 3C).

\section{Expressions of cardiac-specific transcription factors and EMT-associated genes in different cell groups after the induction of MSCs differentiation}

The expressions of cardiac-specific transcription factors including Mesp1, Nkx2.5, Gata4, Gata6, and Isl1 were examined at different time points after the induction of cardiogenic differentiation. They all showed remarkably higher expressions in the lncRNA-Bvht group than the blank control and null vector control groups both under the normal condition and after hypoxia/reoxygenation $(P<0.01$; Fig. 4). Expression levels of EMT-associated genes including Snail, Twist and $\mathrm{N}$-cadherin were also upregulated in lncRNA-Bvht group compared with the other two groups $(P<0.01$; Fig. 5$)$.

Table 1 List of primers for quantitative real-time polymerase chain reaction

\begin{tabular}{|c|c|c|}
\hline Genes & Forward & Reverse \\
\hline cTnT & 5'- CAAAGATGCTGAAGAAGGTC-3' & 5'- GATCAGAGTCTGTAGCTCATTC-3' \\
\hline$a-S A$ & 5'- GATTAATGTTGCTGTTACCC-3' & 5'- CCATGCTCTGTGAAATAATC-3' \\
\hline Gata4 & 5'- CAAAGCAGCCTTGGTGACTA-3' & 5'- AGAAAGTCCCAGAGCCAGGTA-3' \\
\hline Gata6 & 5'- TTGCGGGCTCTATATGAAA-3' & 5'- GCTTGTGTAGAAGGAGAAGT-3' \\
\hline $\mathrm{Nk} \times 2.5$ & 5'- CTITAGGAGAAGGGCGATGA-3' & 5'- GGATGGATCGGAGAAAGGT-3' \\
\hline$|s|-1$ & 5'- GCTGTTTCTATATTGGTCAC-3' & 5'- GCTTAAGAGACCCAGAATTT-3' \\
\hline Snail & 5'- TCTGCACGACCTGTGGAAAG-3' & 5'- TTGGAGCGGTCAGCAAAAG-3' \\
\hline Twist & 5'- GAGACTCTGGAGCTGGATAACT-3' & 5'- CACAAACGAGTGTTCAGACTTC-3' \\
\hline N-cadherin & 5'- GTGGGAATCAGACGGCTAGA-3 & 5'- GCTGCCCTCGTAGTCAAAGA-3' \\
\hline Mesp1 & 5'- CAGAAACAGCATCCCAGGAA-3' & 5'- TTCTAGAAGAGCCAGCATGTC-3' \\
\hline$\beta$-actin & 5'-AGGGAAATCGTGCGTGACAT-3' & 5'-GAACCGCTCATTGCCGATAG-3' \\
\hline
\end{tabular}




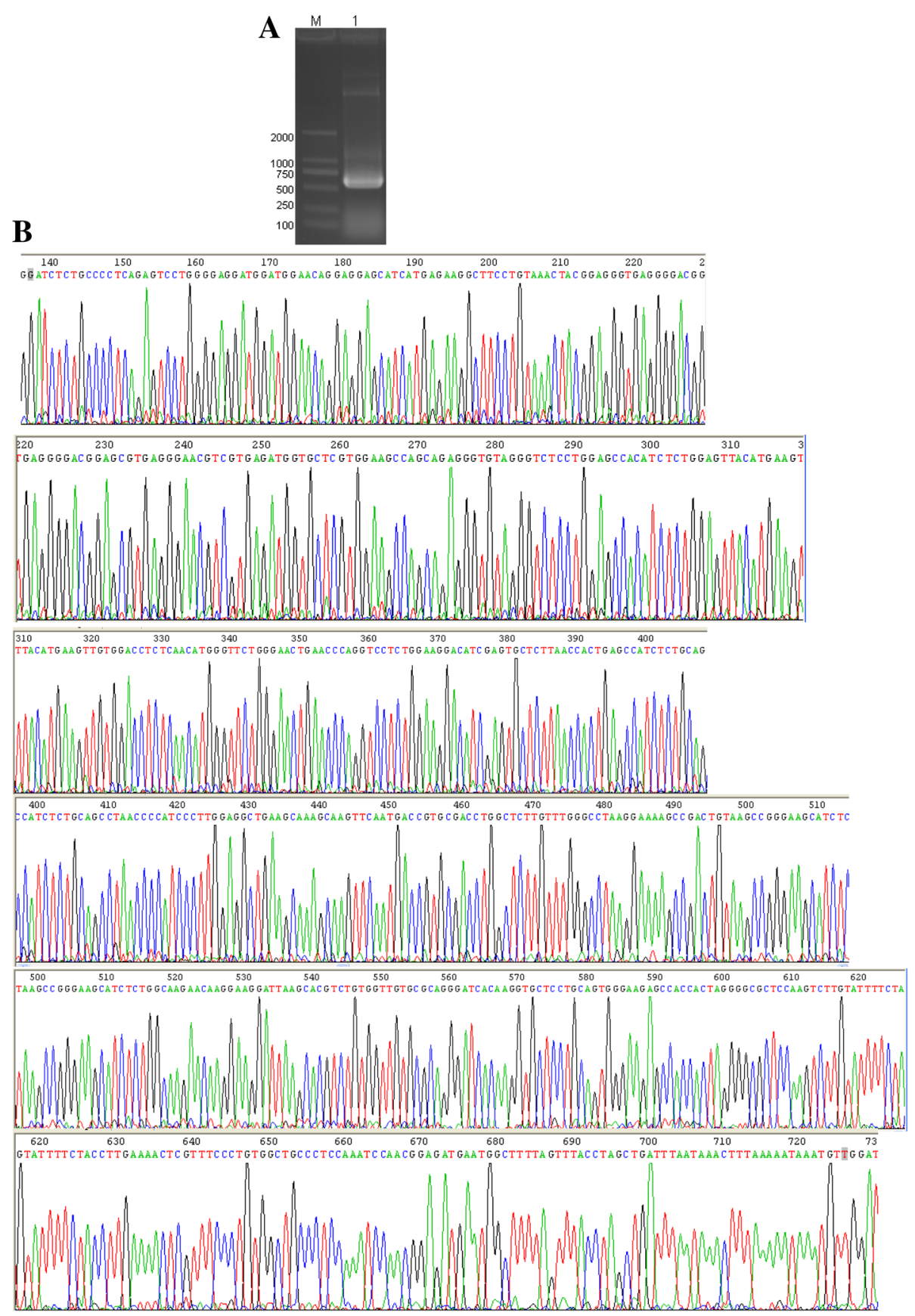

Fig. 1 Electrophoresis of PCR products and sequence analysis of IncRNA-Bvht. A showed the specific band of IncRNA-Bvht at 500 bp by electrophoresis; (B) showed that the IncRNA-Bvht sequence was correctly constructed

Inhibition of Mesp1 interfered with MSCs transdifferentiation into cells with the cardiogenic phenotype induced by IncRNA-Bvht

Anti-Mesp1 siRNAs and control siRNAs (NC) were transiently transfected into undifferentiated MSCs before lncRNA-Bvht transfection and further induction of cardiogenic differentiation. Expressions of Mesp1 and relevant downstream molecules were analyzed $72 \mathrm{~h}$ later.
A significant reduction in Mesp1 expression was observed in the anti-Mesp1 siRNA group. Expressions of cardiac differentiation-associated genes including Nkx2.5, Gata4, Gata6, and Isl1 were all decreased, and EMT-associated genes including Snail, Twist and N-cadherin were downregulated under the condition of Mesp1 inhibition in the lncRNA-Bvht transfection group both under normoxia and after hypoxia/reoxygenation $(P<0.01$; Fig. 6$)$. 


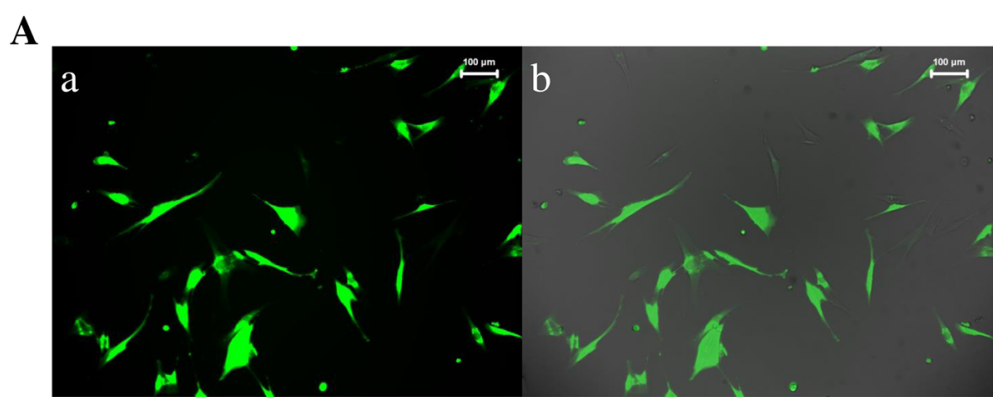

$\mathbf{B}$

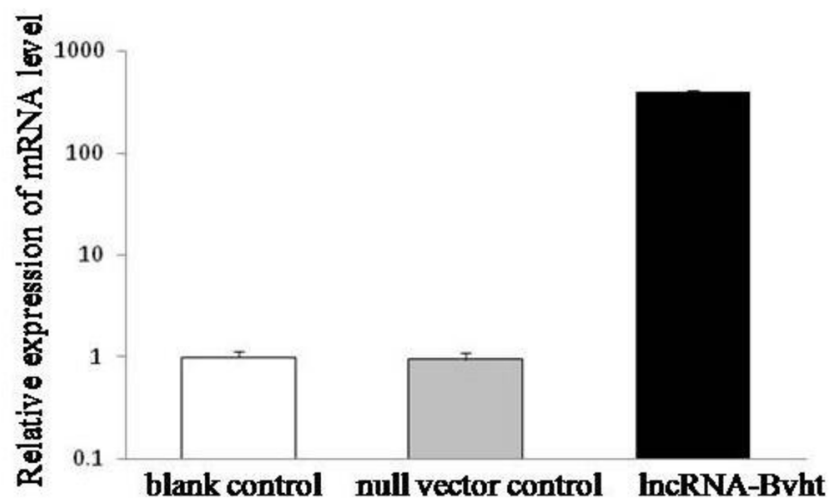

Fig. 2 Detection of IncRNA-Bvht transfection efficiency. IncRNA-Bvht transfection efficiency was detected by the expression of ZsGreen and mRNA level of IncRNA-Bvht. A MSCs expressing ZsGreen after IncRNA-Bvht transfection were shown by fluorescent microscopy ( $\times 400$ ); a represented MSCs transfected with IncRNA-Bvht and $\mathbf{b}$ showed that all the cells with ZsGreen expression were obtained. B The expression of IncRNA-Bvht in different cell groups was detected by quantitative real-time PCR

\section{Discussion}

This study demonstrated that lncRNA-Bvht tranfection could efficiently promote MSCs transdifferentiation into cells with the cardiogenic phenotype in vitro. MSCsderived cells expressed cardiac-specific markers including $c \operatorname{Tn} T$ and $\alpha$-SA. Cardiac-specific transcription factors and EMT-associated genes were upregulated following lncRNA-Bvht transfection both under normal condition and after hypoxia/reoxygenation. However, the expressions of these molecules all presented a converse alteration under the condition of Mesp1 inhibition prior to lncRNA-Bvht transfection.

To derive cardiomyocytes from stem cell precursors has been adopted as a pivotal therapeutic strategy for the repair of the injured heart. MSCs provide a valuable platform for the treatment of heart disease based on regenerative medicine [22]. Nevertheless, they show limited cardiomyogenic potential in spite of functional benefits resulting from their transplantation. Arduous efforts have been made to escalate the efficiency of cardiogenic differentiation of these cells. However, the efficacy of cellular cardiomyoplasty with MSCs remains frustrating, raising the need for alternative induction methods.

IncRNAs have been shown to be implicated in the modulation of stem cell pluripotency and cardiac differentiation [23]. It is revealed that lncRNAs are integral components of stem cell transcriptional networks $[24,25]$. The knockdown or overexpression of relevant IncRNAs reciprocally influences the pluripotent transcription factors, dominating stem cell pluripotent state and lineage specificity [26-28]. Other studies have exhibited that lncRNAs regulate the cellular reprogramming process and play a pivotal role during the reprogramming of somatic cells $[29,30]$. IncRNAs performing as competitive endogenous RNAs (ceRNAs) have been found to be differentially expressed in differentiating human cardiac progenitor cells (CPCs). These ceRNAs exert regulatory roles in cardiac lineage specification and differentiation [31].

Much attention has been drawn to the role of IncRNAs in heart development and cardiac differentiation [32]. Several lncRNAs have been uncovered as critical players in the development of the early cardiovascular system and cardiac differentiation [13, 14]. Some enhancer-associated lncRNAs have also been reported to take control of cardiac specification, differentiation and homeostasis [33]. IncRNA-Bvht is a newly discovered cardiac-specific lncRNA in the mouse. It promotes cardiogenic differentiation of ESCs and retains the cardiac phenotype in neonatal cardiomyocytes [14]. In this study, lncRNA-Bvht was transfected into MSCs in order to 


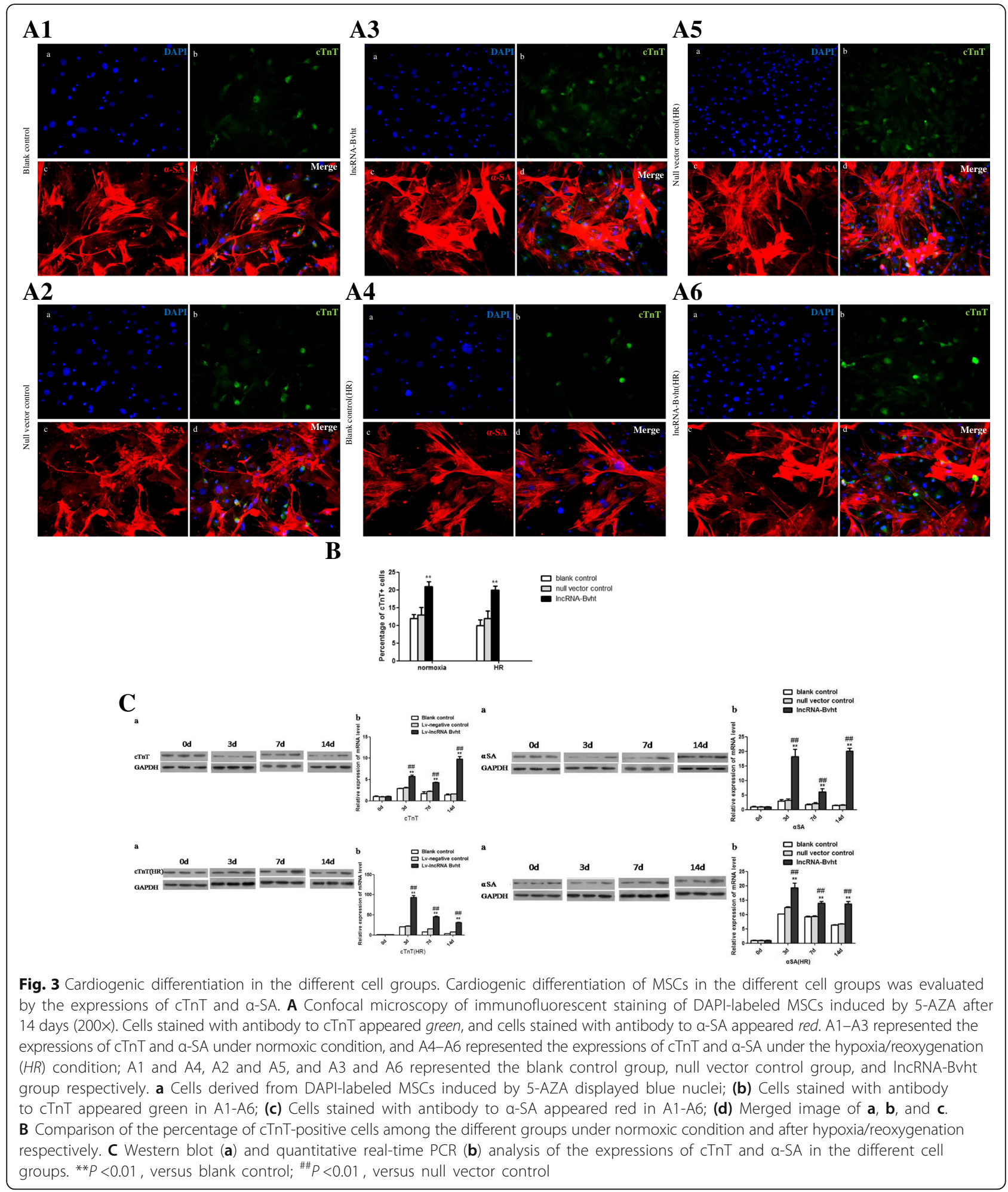

investigate its effect on cardiogenic differentiation of these cells. We discovered that a larger proportion of cells with the cardiogenic phenotype were induced after IncRNABvht transfection. IncRNA-Bvht transfected MSCs displayed evenly distributed and regularly organized myofibrils after 14 days of stimuli in culture. The differentiated cells were shown to have a mature cardiogenic phenotype as evidenced by a much higher expression of cTnT. There was an enhanced level of cardiac-specific transcription factors incuding Nkx2.5, Gata4, Gata6, and 

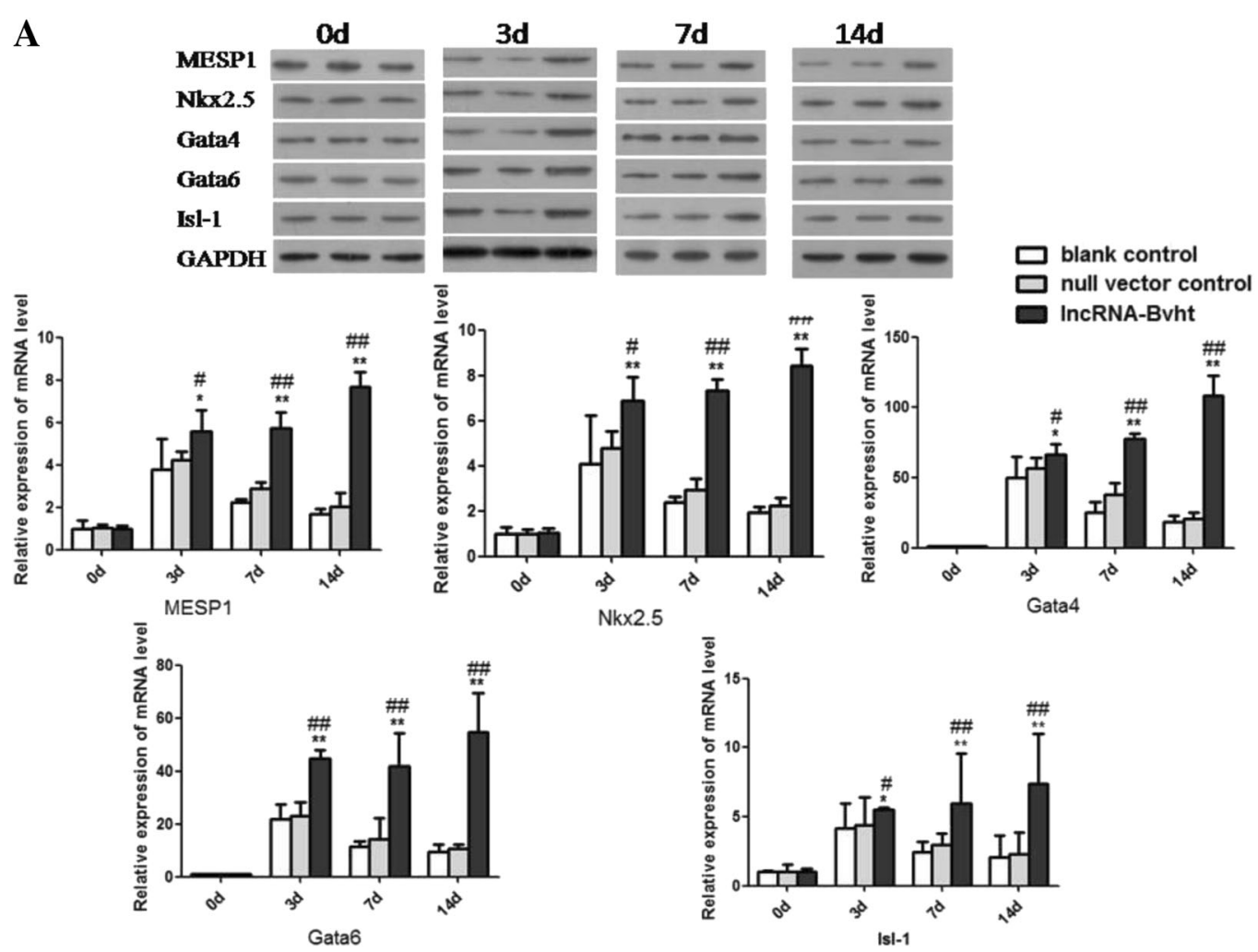

B
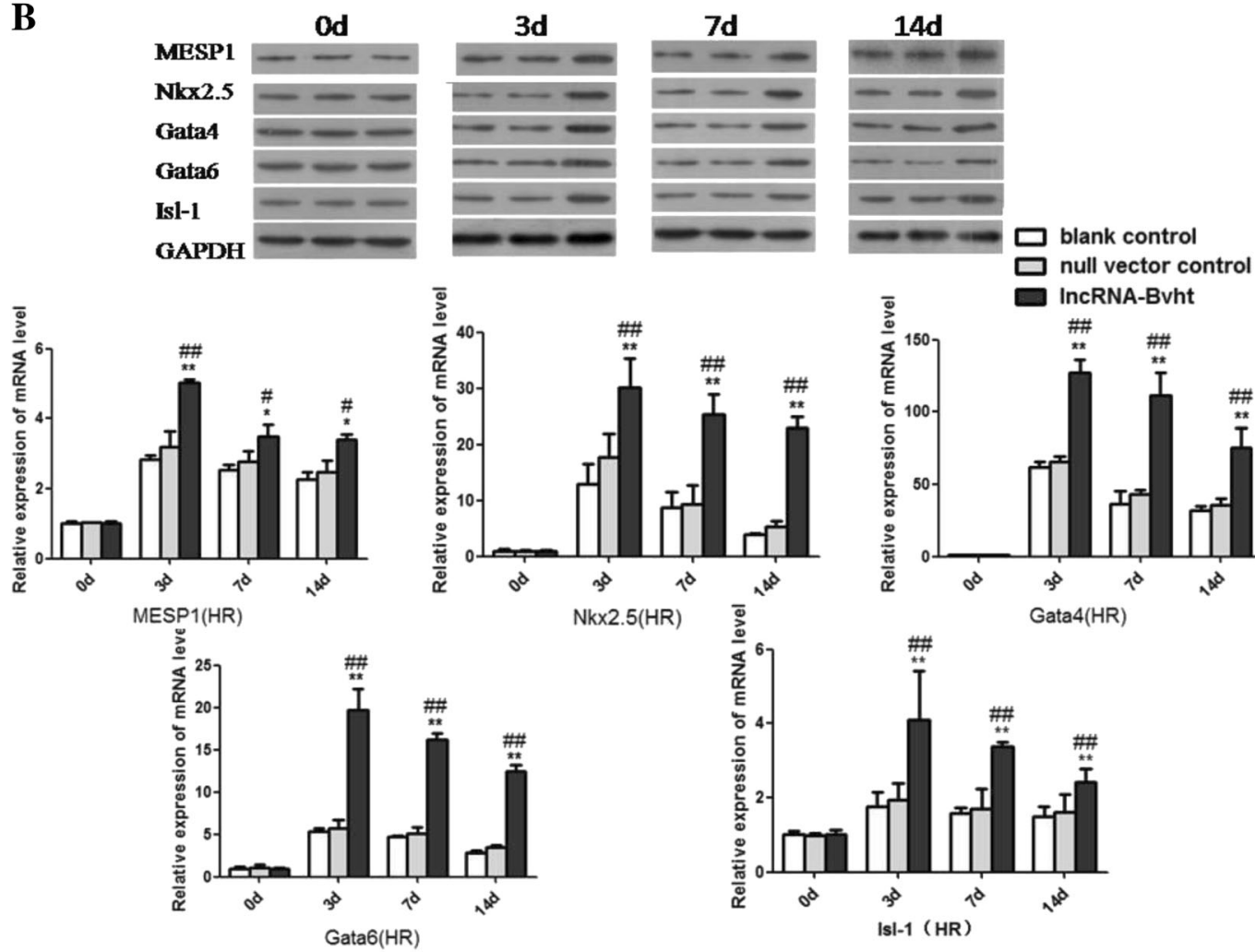

Fig. 4 Expressions of cardiac-specific transcription factors in different cell groups after the induction of cardiogenic differentiation. A Expressions of cardiac-specific transcription factors in different cell groups under normoxic condition. B Expressions of cardiac-specific transcription factors in different cell groups after hypoxia/reoxygenation (HR). ${ }^{*} P<0.05$, ${ }^{*} P<0.01$, vesus blank control; \# $P<0.05$, \#\# $P<0.01$, vesus null vector control 


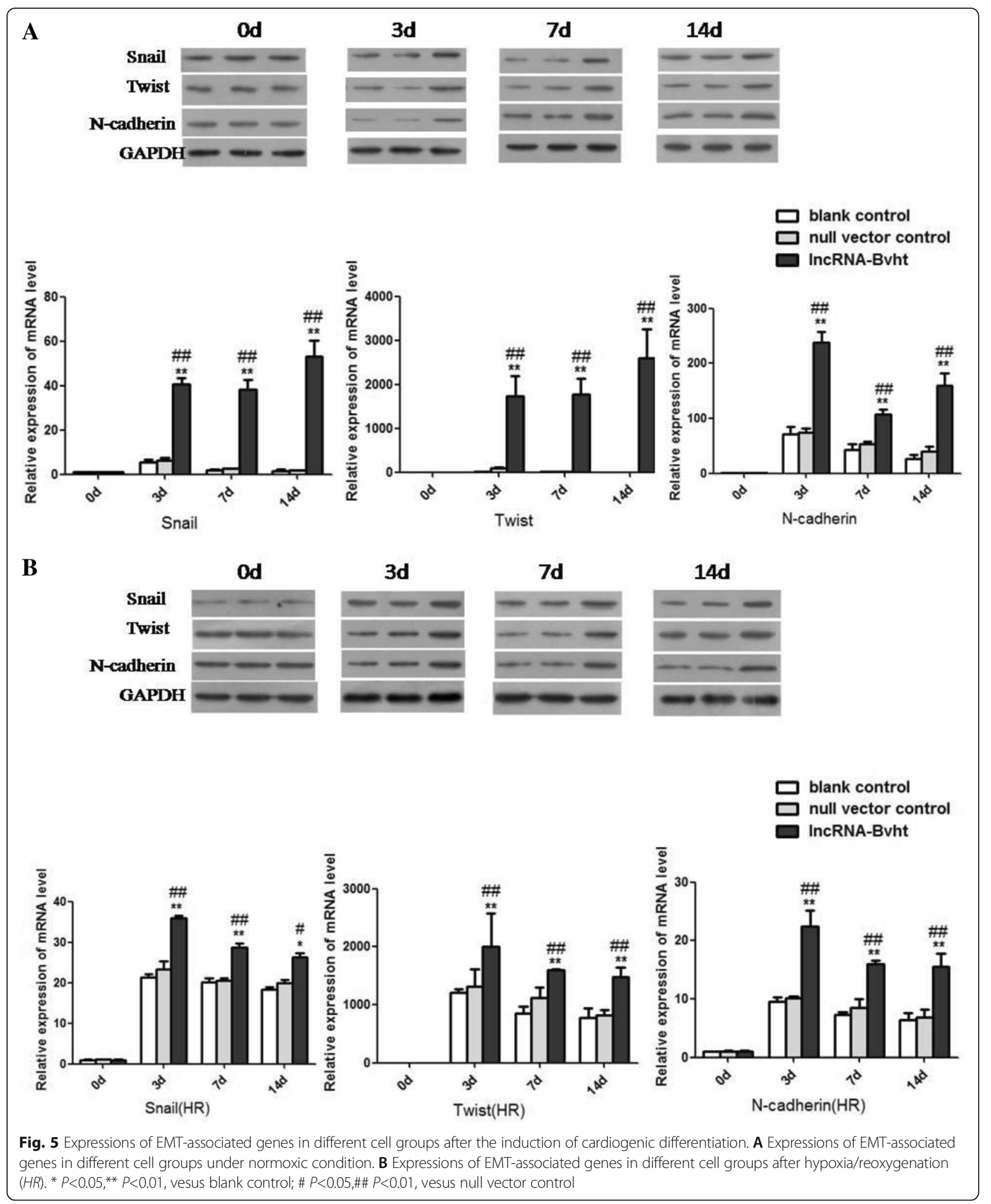




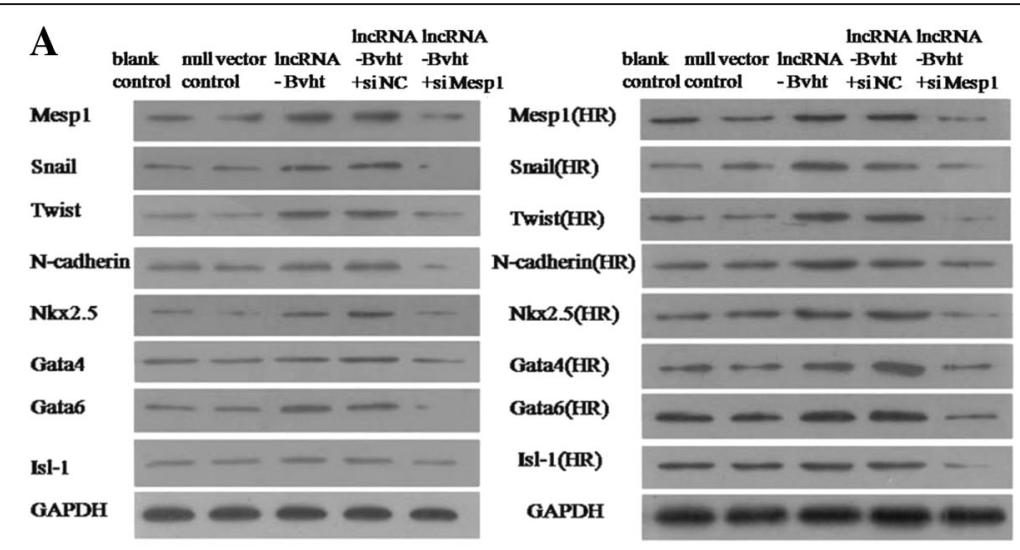

B
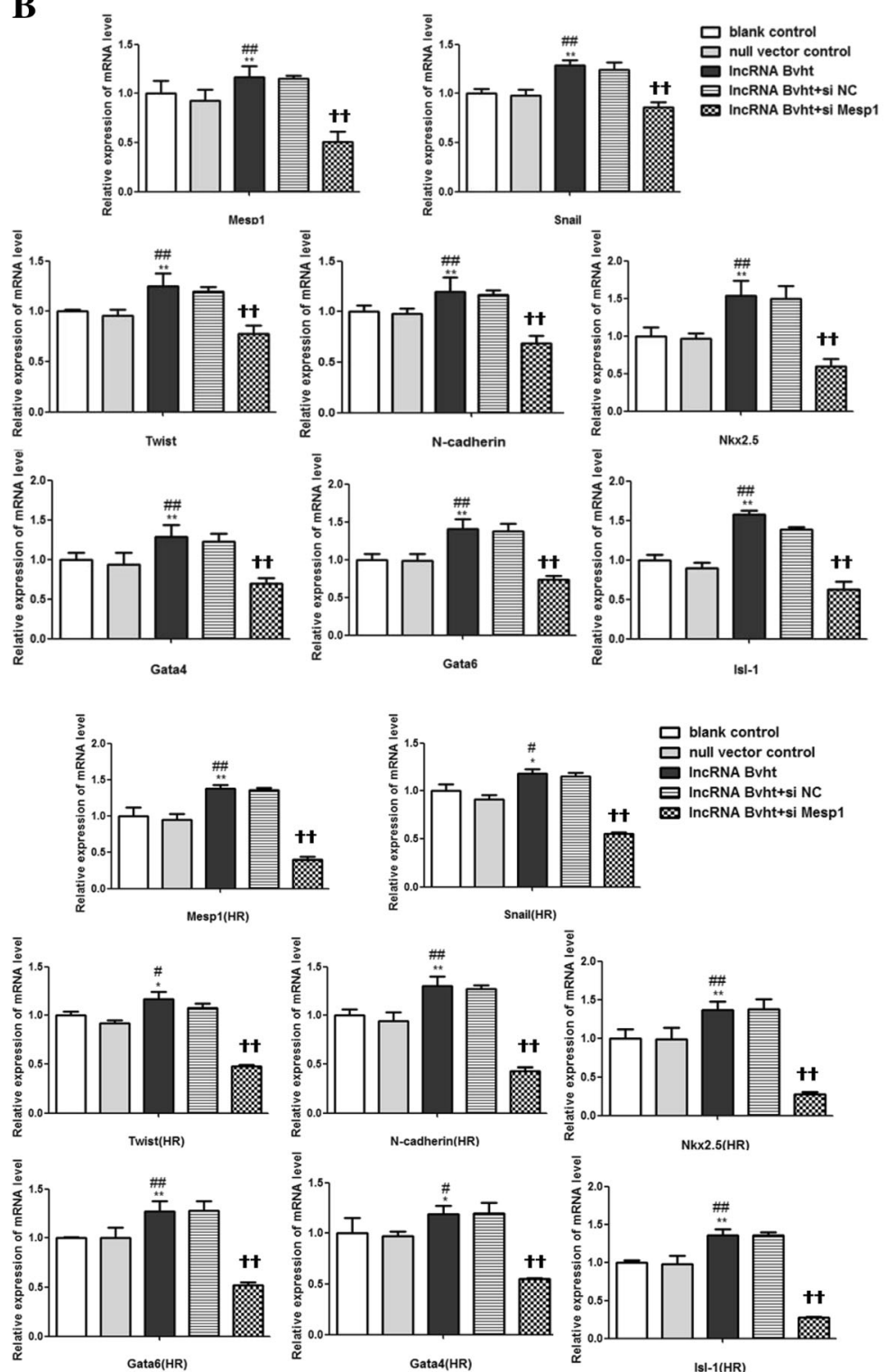

Isl-1

Fig. 6 Expressions of cardiac-specific transcription factors and EMT-associated genes in different cell groups after the inhibition of Mesp1. Western blot (A) and quantitative real-time PCR (B) analysis of cardiac-specific transcription factors and EMT-associated genes in different cell groups after the inhibition of Mesp1. HR hypoxia/reoxygenation. ${ }^{*} P<0.05,{ }^{*} P<0.01$, vesus blank control; $\# P<0.05$, \#\#P<0.01, vesus null vector control; $¥ P<0.05$, $\neq \neq P<0.01$, vesus IncRNA-Bvht 
Isl-1, suggesting that lncRNA-Bvht could efficiently induce the transdifferentiation of MSCs into cells with the cardiogenic phenotype in vitro by promoting the expressions of cardiac differentiation-associated genes.

Studies have demonstrated that EMT exerts a crucial role during heart development and cardiac repair. It facilitates CPCs formation during embryonic development and leads to the generation of CPCs in the adult heart [34]. EMT positively regulates cardiac differentiation of ESCs and PSCs $[17,18]$. Further investigations into the EMT-based regenerative program might contribute to a more efficient strategy for cardiomyogenesis of stem cells and cardiac repair. In this study, genes implicated in EMT such as Snail and Twist were upregulated during lncRNA-Bvht induced transdifferentiation of MSCs. Twist is a crucial transcription factor that participates in the regulation of multi-progenitor states and lineage specificity of MSCs [35, 36]. It has recently been reported that Twist can modulate MSCs lineage commitment by effecting MSCs fate switching between osteogenesis and adipogenesis [36]. In this study, Twist was upregulated after lncRNA-Bvht transfaection, indicating that it might facilitate the cardiac lineage differentiation of MSCs. N-cadherin is identified as another indicator of EMT. The EMT process accompanies an elevated expression of $\mathrm{N}$-cadherin. It has been revealed that $\mathrm{N}$-cadherin could be a novel prospective cell surface marker of MSCs that present an apparently higher ability for cardiomyocyte differentiation [37]. N-cadherin has also been shown to be the only cell-surface gene that is highly expressed in the optimal human umbilical cord blood-derived MSCs (hUCB-MSCs). hUCBMSCs with a notable expression of $\mathrm{N}$-cadherin exhibit an enhanced cell survival rate and interact with host cardiomyocytes more effectively [38]. In this study, we also found that $\mathrm{N}$-cadherin expression level was significantly enhanced after lncRNA-Bvht transfection. The aforementioned data suggest that EMT-associated genes were involved in lncRNA-Bvht mediated cardiogenic differentiation of MSCs.

Mesp1 is a basic helix-loop-helix transcription factor that symbolizes the earliest progenitors of the cardiovascular system [19]. Mesp1 positive cells have been reported to show an increased expression of mesodermal and EMT markers and preferential cardiac differentiation, implying a critical role of Mesp1 in promoting EMT and cardiac differentiation [20]. Evidence shows that Mesp1 regulates EMT and cardiovascular differentiation in ESCs and CPCs [20,39]. Several pharmacological agents have been introduced to enhance the cardiomyogenic ability of MSCs for cardiac repair [40]. MSCs treated with these agents show an appreciably elevated expression of Mesp1, with a later widespread upregulation of the structural genes of cardiomyocytes. Other data display that a recombinant method using various growth factors and cell cytokines guides MSCs into a cardiac progenitor phenotype with a significantly increased expression of Mesp1, conferring cardiac competency on these cells to regenerate new cardiomyocytes [41]. In this study, Mesp1 was remarkably increased at different time points after the induction of cardiogenic differentiation both under the normal condition and after hypoxia/reoxygenation following the lncRNA-Bvht transfection. There was a distinct upregualtion of cardiac differentiation-associated genes and biomarkers of the EMT process. Mesp1 repression before lncRNA-Bvht transfection significantly downregulated the expressions of cardiac differentiation-associated genes and biomarkers of EMT, indicating that lncRNABvht mediated upregulation of Mesp1 might contribute to the expression of cardiac differentiation-associated genes and biomarkers of the EMT process, which subsequently leads to the transdifferentiation of MSCs into cells with the cardiogenic phenotype.

\section{Limitations of the study}

This study was performed in vitro, in vivo studies will be conducted in the future to investigate the effects of lncRNA-Bvht on cardiomyocyte differentiation of MSCs in vivo. In this work, we mainly demonstrated that lncRNA-Bvht could induce the transdifferentiation of MSCs into cells with cardiogenenic phenotypes. Further studies will be focused on functional properties of these differentiated cells, including their contraction and electrophysiological characteristics and whether they possess the ability to form functional syncytium with host cardiomyocytes. Specific signaling pathways involved in the functional mechanisms of lncRNA-Bvht will also be explored later on.

\section{Conclusions}

lncRNA-Bvht could efficiently promote MSCs transdifferentiation into cells with the cardiogenic phenotype in vitro. It might function via enhancing the expressions of cardiac-specific transcription factors and EMTassociated genes. Mesp1 could be a crucial intermediary in the procedure. This study provides new insight for the application of lncRNAs in stem cell-mediated cardiomyocyte regeneration. Further work on the functional mechanisms of these novel molecules might be conducive for improving the therapeutic efficiency of MSCs transplantation.

\section{Abbreviations}

ceRNA: Competitive endogenous RNA; CPC: Cardiac progenitor cell; EMT: Epithelial-mesenchymal transition; ESC: Embryonic stem cell; hUCB-MSC: Human umbilical cord blood-derived mesenchymal stem cell; IncRNA-Bvht: long noncoding RNA Braveheart; IncRNA: long noncoding RNA; Mesp1: Mesoderm posterior 1; MSC: Mesenchymal stem cell; ncRNA: noncoding RNA; PBS: Phosphate-buffered saline; PSC: Pluripotent stem cell 


\section{Acknowledgments}

This work was performed by the investigators of the Sun Yat-sen Memorial Hospital of Sun Yat-sen University. The authors thank Shaoxin Zheng for special technical assistance in the experiments. The authors take responsibility for all aspects of the work and have no differences with regard to the data presentation and interpretation.

\section{Funding}

This study was supported by National Natural Science Foundation of China (Nos. 81070125, 81270213, and 81670306), Science and Technology Foundation in Guangdong Province (Nos. 2010B031600032 and 2014A020211002), the Fundamental Research Funds for the Central Universities (No. 13ykzd16), and the Medical Science and Technology Research Fund of Guangdong Province (No. A2016264).

\section{Availability of supporting data}

All data generated or analyzed during this study are included in this published article.

\section{Authors' contributions}

$\mathrm{JH}$ and $\mathrm{HL}$ carried out the cell culture, transfection, participated in the statistical analysis, and drafted the manuscript. CZ and HW carried out the molecular assay. TG participated in the vector construction. QW and TZ carried out cell staining and statistical analysis. SZ participated with technical assistance. TW conceived the study and participated in the study design. All authors read and approved the final manuscript.

\section{Competing interests}

The authors declare that they have no competing interests.

\section{Consent for publication}

Not applicable.

\section{Ethical approval and consent to participate}

Animals were obtained from the Animal Experimental Center of the Sun Yat-sen University. All animal handling and procedures were performed in accordance with protocols approved by the Animal Ethics Committee of Sun Yat-sen University (201210016).

\section{Author details}

${ }^{1}$ Guangdong Provincial Key Laboratory of Malignant Tumor Epigenetics and Gene Regulation, the Sun Yat-sen Memorial Hospital of Sun Yat-sen University, 107 Yanjiang Xi Road, Guangzhou, Guangdong 510120, China. ${ }^{2}$ Guangdong Province Key Laboratory of Arrhythmia and Electrophysiology, 107 Yanjiang Xi Road, Guangzhou, Guangdong 510120, China. ${ }^{3}$ Department of Emergency, the Sun Yat-sen Memorial Hospital of Sun Yat-sen University, 107 Yanjiang Xi Road, Guangzhou, Guangdong 510120, China.

\section{Received: 9 September 2016 Revised: 11 November 2016}

Accepted: 8 December 2016 Published online: 17 January 2017

\section{References}

1. Mozaffarian D, Benjamin EJ, Go AS, Arnett DK, Blaha MJ, Cushman M, et al. Heart disease and stroke statistics-2015 update: a report from the American Heart Association. Circulation. 2015;131(4):e29-322.

2. Hou J, Wang L, Jiang J, Zhou C, Guo T, Zheng S, et al. Cardiac stem cells and their roles in myocardial infarction. Stem Cell Rev. 2013;9(3):326-38.

3. Fox IJ, Daley GQ, Goldman SA, Huard J, Kamp TJ, Trucco M. Stem cell therapy. Use of differentiated pluripotent stem cells as replacement therapy for treating disease. Science. 2014;345:1247391.

4. Garbern JC, Lee RT. Cardiac stem cell therapy and the promise of heart regeneration. Cell Stem Cell. 2013;12:689-98.

5. Hou J, Wang L, Hou J, Guo T, Xing Y, Zheng S, et al. Peroxisome proliferator-activated receptor gamma promotes mesenchymal stem cells to express connexin43 via the inhibition of TGF- $\beta 1 /$ Smads signaling in a rat model of myocardial infarction. Stem Cell Rev. 2015;11(6):885-99

6. Piryaei A, Soleimani M, Heidari MH, Saheli M, Rohani R, Almasieh M. Ultrastructural maturation of human bone marrow mesenchymal stem cells-derived cardiomyocytes under alternative induction of 5-azacytidine. Cell Biol Int. 2015;39(5):519-30.
7. Xing $Y, L v A$, Wang $L$, Yan $X$. The combination of angiotensin II and 5-azacytidine promotes cardiomyocyte differentiation of rat bone marrow mesenchymal stem cells. Mol Cell Biochem. 2012;360(1-2):279-87.

8. Perán M, López-Ruiz E, González-Herrera L, Bustamante M, Valenzuela A Marchal JA. Cellular extracts from post-mortem human cardiac tissue direct cardiomyogenic differentiation of human adipose tissue-derived stem cells. Cytotherapy. 2013;15(12):1541-8.

9. Wamstad JA, Wang X, Demuren OO, Boyer LA. Distal enhancers: new insights into heart development and disease. Trends Cell Biol. 2014;24:294-302.

10. Lam MT, Li W, Rosenfeld MG, Glass CK. Enhancer RNAs and regulated transcriptional programs. Trends Biochem Sci. 2014;39:170-82.

11. Beilharz TH. Understanding the regulation of coding and noncoding transcription in cell populations. Curr Genet. 2016;62(2):317-9.

12. Dey BK, Mueller AC, Dutta A. Long non-coding RNAs as emerging regulators of differentiation, development, and disease. Transcription. 2014:5(4), e944014

13. Grote P, Wittler L, Hendrix D, Koch F, Währisch S, Beisaw A, et al. The tissue-specific IncRNA Fendrr is an essential regulator of heart and body wall development in the mouse. Dev Cell. 2013;24:206-14.

14. Klattenhoff CA, Scheuermann JC, Surface LE, Bradley RK, Fields PA Steinhauser ML, et al. Braveheart, a long noncoding RNA required for cardiovascular lineage commitment. Cell. 2013;152:570-83.

15. Pasquier J, Abu-Kaoud NA, Thani H, Rafii A. Epithelial to mesenchymal transition in a clinical perspective. J Oncol. 2015;2015:792182.

16. Asli NS, Harvey RP. Epithelial to mesenchymal transition as a portal to stem cell characters embedded in gene networks. Bioessays. 2013;35(3):191-200.

17. Richter A, Valdimarsdottir L, Hrafnkelsdottir HE, Runarsson JF, Omarsdottir AR, Ward-van Oostwaard D, et al. BMP4 promotes EMT and mesodermal commitment in human embryonic stem cells via SLUG and MSX2. Stem Cells. 2014;32(3):636-48.

18. Zhang J, Klos M, Wilson GF, Herman AM, Lian X, Raval KK, et al. Extracellular matrix promotes highly efficient cardiac differentiation of human pluripotent stem cells: the matrix sandwich method. Circ Res. 2012;111(9):1125-36.

19. David R, Schwarz F, Rimmbach C, Nathan P, Jung J, Brenner C, et al. Selection of a common multipotent cardiovascular stem cell using the 3.4-kb Mesp1 promoter fragment. Basic Res Cardiol. 2013;108(1):312.

20. Den Hartogh SC, Schreurs C, Monshouwer-Kloots JJ, Davis RP, Elliott DA, Mummery $\mathrm{CL}$, et al. Dual reporter MESP1 mCherry/w-NKX2-5 eGFP/w hESCs enable studying early human cardiac differentiation. Stem Cells. 2015;33(1):56-67.

21. Wang T, Tang W, Sun S, Ristagno G, Huang Z, Weil MH. Intravenous infusion of bone marrow mesenchymal stem cells improves myocardial function in a rat model of myocardial ischemia. Crit Care Med. 2007:35:2587-93.

22. Xing Y, Hou J, Guo T, Zheng S, Zhou C, Huang H, et al. microRNA-378 promotes mesenchymal stem cell survival and vascularization under hypoxic-ischemic conditions in vitro. Stem Cell Res Ther. 2014;5(6):130.

23. Kim DH, Marinov GK, Pepke S, Singer ZS, He P, Williams B, et al. Single-cell transcriptome analysis reveals dynamic changes in IncRNA expression during reprogramming. Cell Stem Cell. 2015;16(1):88-101.

24. Savić N, Bär D, Leone S, Frommel SC, Weber FA, Vollenweider E, et al. IncRNA maturation to initiate heterochromatin formation in the nucleolus is required for exit from pluripotency in ESCs. Cell Stem Cell. 2014;15(6):720-34.

25. Li YP, Wang Y. Large noncoding RNAs are promising regulators in embryonic stem cells. J Genet Genomics. 2015;42(3):99-105.

26. Schonrock N, Harvey RP, Mattick JS. Long noncoding RNAs in cardiac development and pathophysiology. Circ Res. 2012;111:1349-62.

27. Flynn RA, Chang HY. Long noncoding RNAs in cell-fate programming and reprogramming. Cell Stem Cell. 2014;14(6):752-61.

28. Ghosal S, Das S, Chakrabarti J. Long noncoding RNAs: new players in the molecular mechanism for maintenance and differentiation of pluripotent stem cells. Stem Cells Dev. 2013:22(16):2240-53.

29. Bao X, Wu H, Zhu X, Guo X, Hutchins AP, Luo Z, et al. The p53-induced lincRNA-p21 derails somatic cell reprogramming by sustaining H3K9me3 and CpG methylation at pluripotency gene promoters. Cell Res. 2015:25(1):80-92.

30. Sadahiro T, Yamanaka S, leda M. Direct cardiac reprogramming: progress and challenges in basic biology and clinical applications. Circ Res. 2015;116(8):1378-91. 
31. Ounzain S, Pezzuto I, Micheletti R, Burdet F, Sheta R, Nemir M, et al. Functional importance of cardiac enhancer-associated noncoding RNAs in heart development and disease. J Mol Cell Cardiol. 2014;76:55-70.

32. Ounzain S, Crippa S, Pedrazzini T. Small and long non-coding RNAs in cardiac homeostasis and regeneration. Biochim Biophys Acta. 2013;1833(4):923-33.

33. Ounzain S, Micheletti R, Arnan C, Plaisance I, Cecchi D, Schroen B, et al. CARMEN, a human super enhancer-associated long noncoding RNA controlling cardiac specification, differentiation and homeostasis. J Mol Cell Cardiol. 2015;89(Pt A):98-112.

34. Limana F, Bertolami C, Mangoni A, Di Carlo A, Avitabile D, Mocini D, et al. Myocardial infarction induces embryonic reprogramming of epicardial c-kit(+) cells: role of the pericardial fluid. J Mol Cell Cardiol. 2010;48:609-18.

35. Boregowda SV, Krishnappa V, Haga CL, Ortiz LA, Phinney DG. A clinical indications prediction scale based on TWIST1 for human mesenchymal stem cells. EBioMedicine. 2015;4:62-73.

36. Camp E, Anderson PJ, Zannettino AC, Gronthos S. Tyrosine kinase receptor c-ros-oncogene 1 mediates TWIST-1 regulation of human mesenchymal stem cell lineage commitment. Bone. 2016;94:98-107.

37. Ishimine H, Yamakawa N, Sasao M. N-Cadherin is a prospective cell surface marker of human mesenchymal stem cells that have high ability for cardiomyocyte differentiation. Biochem Biophys Res Commun. 2013;438(4):753-9.

38. Lee EJ, Choi EK, Kang SK, Kim GH, Park JY, Kang HJ, et al. N-cadherin determines individual variations in the therapeutic efficacy of human umbilical cord blood-derived mesenchymal stem cells in a rat model of myocardial infarction. Mol Ther. 2012;20(1):155-67.

39. Chiapparo G, Lin X, Lescroart F. Mesp1 controls the speed, polarity, and directionality of cardiovascular progenitor migration. J Cell Biol. 2016;213(4):463-77.

40. Yang J, Kaur K, Ong LL, Eisenberg CA, Eisenberg LM. Inhibition of G9a histone methyltransferase converts bone marrow mesenchymal stem cells to cardiac competent progenitors. Stem Cells Int. 2015;2015:270428.

41. Behfar A, Yamada S, Crespo-Diaz R, Nesbitt JJ, Rowe LA, Perez-Terzic C, et al. Guided cardiopoiesis enhances therapeutic benefit of bone marrow human mesenchymal stem cells in chronic myocardial infarction. J Am Coll Cardiol. 2010;56(9):721-34

\section{Submit your next manuscript to BioMed Central and we will help you at every step:}

- We accept pre-submission inquiries

- Our selector tool helps you to find the most relevant journal

- We provide round the clock customer support

- Convenient online submission

- Thorough peer review

- Inclusion in PubMed and all major indexing services

- Maximum visibility for your research

Submit your manuscript at www.biomedcentral.com/submit

) Biomed Central 